\title{
Simulations of the rf heating rates in a linear quadrupole ion trap
}

\author{
Vladimir L. Ryjkov, ${ }^{*}$ XianZhen Zhao, ${ }^{\dagger}$ and Hans A. Schuessler \\ Department of Physics, Texas A \& M University, College Station, Texas 77843, USA
}

(Received 24 January 2005; published 23 March 2005)

\begin{abstract}
Molecular dynamics simulations of the ion clouds stored in a linear quadrupole rf ion trap are performed at low temperatures $(0.1 \mathrm{mK}-15 \mathrm{~K})$. The introduction of periodic boundary conditions allowed us to study the translationally uniform ion cloud geometry. The time evolution of the kinetic energy of the ion cloud is monitored during the simulation and the increase in the kinetic energy of the cloud (rf heating) is determined; its dependence on temperature, trapping voltage, and the number of ions is studied. The dependence of $\mathrm{rf}$ heating rate on temperature shows that $\mathrm{rf}$ heating is undetectable until the temperature reaches approximately $0.5 \mathrm{~K}$; it then increases rapidly until it reaches a maximum at around $2 \mathrm{~K}$. It starts to decrease slowly at higher temperatures. The $\mathrm{rf}$ heating rate is shown to increase very sharply with the amplitude of the trapping voltage. The dependence of the rf heating rate on the number of ions shows the influence of the ion crystal shell structure at low temperatures, and has a simple linear dependence at higher temperatures.
\end{abstract}

DOI: $10.1103 /$ PhysRevA.71.033414

PACS number(s): 32.80.Pj, 52.25.-b, 52.65.Yy

In $\mathrm{rf}$ ion traps the confinement of ions is achieved by the ponderomotive effect of a fast-alternating quadrupole potential [1]. When applied to many ions, such a rf potential increases the kinetic energy of the ions ( $\mathrm{rf}$ heating). Application of laser cooling to the stored ions can overcome this heating effect, and achieve milliKelvin-range temperatures. Previous research efforts [2,3] have identified some of the important properties of $\mathrm{rf}$ heating. However, a more detailed understanding of the rf heating is needed to study its effects in large ion clouds $[4,5]$. This effect is also important in areas such as sympathetic cooling $[6,7]$, and for crystalline beams [8].

As the number of ions in the cloud grows, a thermodynamics approach to interpreting the ion cloud dynamics becomes more appropriate. We use the molecular dynamics (MD) method to study the rates with which the kinetic energy (temperature) increases. Applying molecular dynamics methods to the non-neutral plasma confined in an ion trap meets with extra difficulties as compared to the traditional liquid or solid state simulations. First, the Coulomb interaction between the particles in the plasma has a theoretically unlimited range. Therefore it is impossible to limit the interactions between the particles to just a few neighbors. Ideally one would have to account for interactions between each pair of particles in the system. Second, in addition to the Coulomb interaction between the particles there is an external oscillating potential. This introduces many different time scales into the picture, such as the time scale of the ion's secular oscillation $\left(10^{-5} \mathrm{~s}\right)$, and the time scale of the particle motion in the oscillating potential $\left(10^{-7} \mathrm{~s}\right)$. A time step has to be chosen so that it is small on the fastest time scale, yet in order to deduce any meaningful conclusions the simulation has to continue long enough even on the slowest time scale. All this makes the molecular dynamics simulations of the ion

\footnotetext{
*Present address: TRIUMF, Vancouver, BC, Canada.

${ }^{\dagger}$ Present address: Dept. of Chemistry, UBC, Vancouver, BC, Canada.
}

trap plasma much more computationally intensive. However, despite the difficulties, the MD approach has been used to obtain the density distribution of the ions in the trap, as well as some dynamic properties of the ion crystals in spherical rf traps [3]. These early simulations have identified some of the important features of the ion cloud dynamics, such as the absence of rf heating at low temperatures. However, the lack of computing power at the time made it impossible to conduct systematic simulations that would monitor the evolution of the cloud over a long period of time and investigate various parameters. A more recent simulation [9] has made progress in that direction, by monitoring the motion of 1000 ions in a spherical ion cloud for 50000 oscillation periods of the trapping field. More important than the scale of that simulation was the fact that it was performed for a range of different temperatures.

In this article we report a different approach to the simulation of long ion clouds in linear ion traps. The rf heating rates in this translationally uniform ion cloud geometry should be easier to describe theoretically. It also allows us to use periodic boundary conditions (PBC's) which significantly reduces the needed computational power. As a result, the simulations were performed to study the rf heating rates for a wider range of temperatures, and the temperature dependence is found to be drastically different from the case of a spherical ion cloud [9]. In addition, the dependence of $\mathrm{rf}$ heating rates on the amplitude of the rf voltage and the size of the ion cloud is studied. Features of the rf heating rates are discussed in this article.

The equations of motion of an ion in a linear quadrupole ion trap are

$$
\frac{d^{2} \vec{r}_{i}}{d t^{2}}=\frac{1}{m_{i}}\left(\vec{F}_{i}^{(T)}+\sum_{j \neq i} k_{C} \frac{Z_{i} Z_{j} e^{2}}{r_{i j}^{2}} \frac{\vec{r}_{i j}}{r_{i j}}\right),
$$

where $m_{i}$ is the mass, and $Z_{i}$ is the charge of the $i$ th ion. The first term in the parentheses, $\vec{F}_{i}^{(T)}$, is the trapping force acting on the ion; the second term is the Coulomb interaction with the other ions stored in the trap. In the radial direction the 


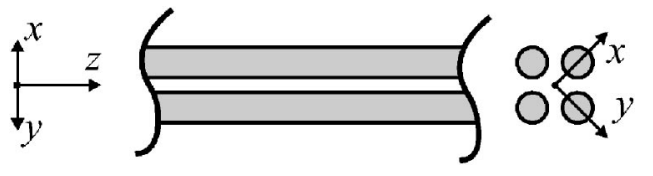

FIG. 1. The geometry of the ion trap and the coordinate system used in this simulation. Trap electrodes extend infinitely along the $z$ axis. The $x$ and $y$ axes point toward the trap electrodes.

trapping force is due to the oscillating quadrupole electric potential:

$$
\Phi^{(T)}(x, y ; t)=\frac{V_{0}}{r_{0}^{2}}\left(x^{2}-y^{2}\right) \cos (\Omega t)
$$

where $V_{0}$ is the amplitude of the rf signal applied to the trap rods, $r_{0}$ is the distance from the center of the trap to the surface of the trap rods, and $\Omega$ is the trapping frequency. Motion of a single particle in such an oscillating harmonic potential is described by the Mathieu equations [10]. The important characteristic value associated with this motion is the $q$ value, $q=4 e V_{0} / m r_{0}^{2} \Omega^{2}$. The time-averaged effect of the rf trapping field can be described by the so-called pseudopotential. For the quadrupole rf trapping field given by Eq. (2) the pseudopotential is a harmonic potential well:

$$
\bar{\Phi}^{(T)}(x, y)=\frac{D}{r_{0}^{2}}\left(x^{2}+y^{2}\right)
$$

where $D=e V_{0}^{2} / m \Omega^{2} r_{0}^{2}$ is the potential depth of the trap. The characteristic frequency of ion oscillations in the harmonic pseudopotential is the secular frequency $\omega_{s}=\sqrt{2} e V_{0} / m \Omega r_{0}^{2}$ $=(\sqrt{2} / 4) q \Omega$.

The solutions for a single particle in a rf field are available analytically and are very well studied [10]. These solutions show that the energy is conserved for a single ion moving in a quadrupole rf field. However, the solution is not valid when many particles interact with each other, as well as with the rf field. The kinetic energy of many ions in a quadrupole rf field increases with time. This well-known effect is called rf heating.

Ion confinement along the trap axis is achieved by applying dc closing voltages to the electrodes at the ends of the trap. In this article the ions are considered to be moving freely along the trap axis. This situation is realized when the distance from the center of the trap to the trap rods $\left(r_{0}\right)$ is much smaller than the length of the trap. This situation matches the geometry of the ion trap that we used in our experiments [11] (the length of the trapping segment is 50 $\mathrm{mm}$, whereas $r_{0}=2.61 \mathrm{~mm}$ ). Many of the parameters of this simulation are chosen so that they match those of our experimental work. The radius of the trapping region $r_{0}$ $=2.61 \mathrm{~mm}$, the frequency of the trapping if field $\Omega=2 \pi$ $\times 7.35 \mathrm{MHz}$, and the ions chosen for the simulation are ${ }^{24} \mathrm{Mg}^{+}$ions $\left(m_{i}=24\right.$ a.u., $\left.Z_{i}=1\right)$.

The coordinates used in the simulation are shown in Fig. 1. We consider an ion cloud that is infinite along the $z$ axis. The advantage of considering an infinite cloud is that by employing the so-called periodic boundary conditions [12], the equations of motion are solved only for a group of ions that are present in a slice of the infinite ion cloud. The PBC's effectively truncate the interaction distance between the ions; therefore the length of the simulated slice has to be larger than the effective interaction distance. Therefore, the length of the slice must be larger than the Debye radius of the confined ion plasma, for the PBC's to produce accurate results.

To solve the equation of motion of the $i$ th particle (1), the Gear algorithm [13] is applied. The time step of the integration is selected so that an even number of integration steps amounts to an oscillation period of the rf trapping field. Typically 200 steps per period were used.

To determine the rate of increase in the kinetic energy due to $\mathrm{rf}$ heating, the simulation is set up in the following way. The ion cloud is prepared in a state that corresponds to the temperature of interest. The kinetic energy is monitored while the cloud is moving under the influence of the rf trapping field and the Coulomb interaction forces. A typical evolution time was $50000 \mathrm{rf}$ oscillation periods. The linear fit to the dependence of the kinetic energy on time determines the rf heating rate.

The radial motion of each ion is a combination of the fast oscillatory micromotion (oscillations with the frequency $\Omega$ of the rf trapping field) and the slow thermal motion (macromotion). One of the important issues of the thermal approach to ion cloud dynamics in a rf field is how to determine the temperature of this complex motion. In this simulation we used the ion velocities at the phase 0 of the rf trapping field to calculate the temperature of the ions. It has been verified that this produces practically the same values of the temperature as an alternative approach $[3,9]$ of using the average velocity value over a rf oscillation period.

All ion cloud configurations used in the simulation were prepared by starting with the ion cloud at ultracold temperature $(T<0.1 \mathrm{mK})$ and heating it up to the desired temperature. In the ultracold state the ions form ordered crystal structures, which consist of cylindrical shells, sometimes with a string of ions along the center. As the number of ions is increased, more shells are formed to accommodate the additional ions. Of interest are the ion crystals for which any additional ions would have to start a new shell; we call these complete-shell ion crystals. For a trapping voltage amplitude $V_{0}=100 \mathrm{~V}$ in a slice of length $L=200 \mu \mathrm{m}$ the complete-shell ion crystals are formed out of $N=68$ (center string + shell) and 186 ions (center string + two shells).

The initial conditions for the higher temperatures $(T$ $>1 \mathrm{mK}$ are of practical interest) are generated by heating up the ions initially prepared in the ultracold state. To do that the velocities are scaled up to match the new higher temperature, and the system is allowed to evolve over $10^{5}$ rf oscillation periods. Since for a wide range of these higher temperatures rf heating is present, the kinetic energy of the ion cloud is rescaled to match the target temperature after each rf oscillation period.

The dependence of the rf heating rate on the temperature of the ion cloud is shown in Fig. 2. Up to $T=5 \mathrm{~K}$ the length of the simulation slice was $L=200 \mu \mathrm{m}$, with the number of ions $N=68$. This corresponds to the complete-shell ion crystal at low temperatures. The graph is extended to larger temperature values by keeping the same linear density of the 


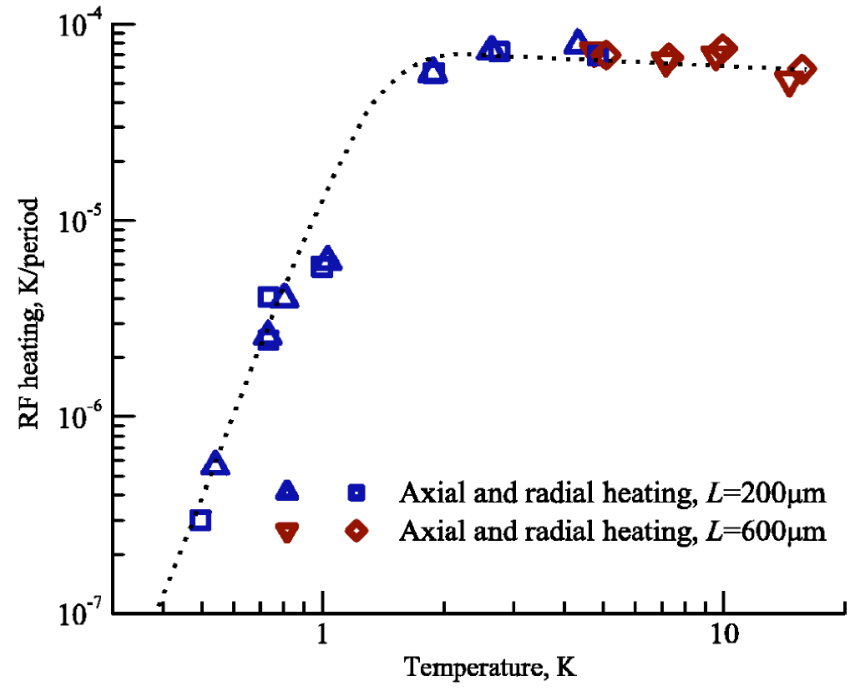

FIG. 2. The $\mathrm{rf}$ heating rate as a function of temperature; trapping voltage amplitude $V_{0}=100 \mathrm{~V}$ (corresponds to $q=0.11$ ). The dashed line is a hand-drawn fit.

ions, but extending the length of the studied slice from $L$ $=200$ to $600 \mu \mathrm{m}$, thus increasing the cutoff length of the Coulomb interactions. The rf heating rate was calculated for both values of $L$ at the temperature $T=5 \mathrm{~K}$ to verify that they produce the same heating rate. The rf heating rate was also verified for several points in the area where it steeply increases by repeating the calculations at $T=0.1,0.5$, and $1 \mathrm{~K}$ with a longer slice length $L=1200 \mu \mathrm{m}$. The fact that the same rf heating is produced for different slice lengths indirectly supports the use of the PBC's. At temperatures below $T=0.5 \mathrm{~K}$, down to a fraction of a millikelvin, no detectable rf heating was observed (we estimate the detection limit of the rf heating rate to be approximately $10 \mathrm{ppb}$ of the temperature per rf oscillation period). This means that it is possible in principle to store the ion clouds in the trap at ultralow temperatures for extended periods of time without involving laser cooling. In practice, however, this possibility is limited by the presence of other heating mechanisms that become important at low ion temperatures, such as heating by the fluctuating patch potentials [14]. rf heating becomes detectable at $T=0.5 \mathrm{~K}$ and rapidly increases in the interval from $T=0.5$ to $2 \mathrm{~K}$. The rf heating rate increases as the fifth power of temperature in this interval. It decreases slowly in the temperature interval from $T=2$ up to $15 \mathrm{~K}$. The rate of this decrease (approximately $T^{-1 / 2}$ ) is much slower compared to the drastic increase in $\mathrm{rf}$ heating at low temperatures. It is important to point out that here we present absolute heating rates, similarly to Ref. [9], and the graphs of temperature dependence appear differently from dimensionless rf heating rates, such as in Ref. [8], which show the fractional temperature increase per rf oscillation period.

An important feature of the temperature dependence presented here is its sharp dependence on the temperature. The simulations of rf heating in a spherical ion cloud [9], as well as the simulation of ion beams in a storage ring [8], show that $\mathrm{rf}$ heating increases as $T^{2}$ at low temperatures. While the similarity of the previously reported data suggests universal

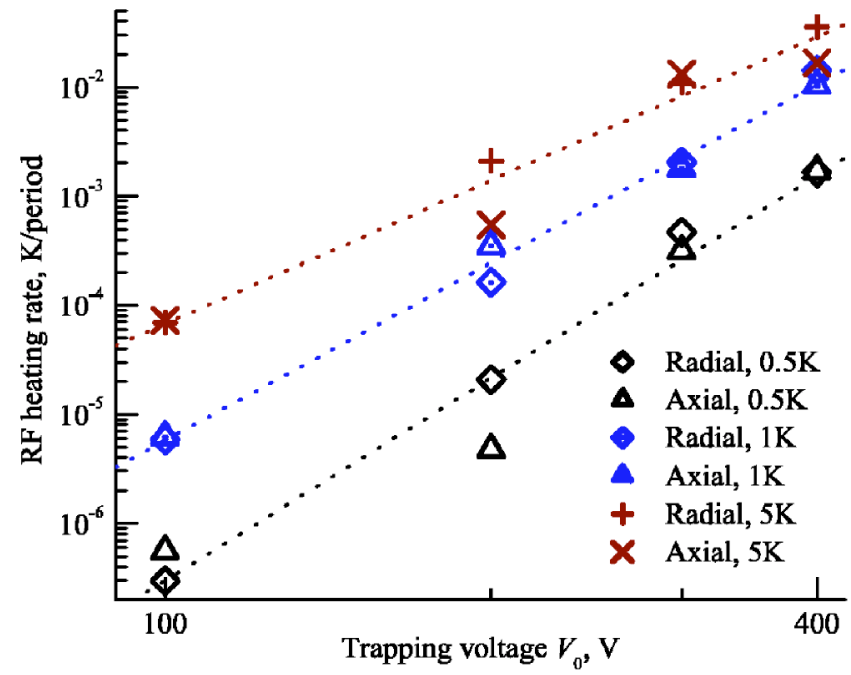

FIG. 3. Change of rf heating rates with trapping voltage for three characteristic ion cloud temperatures $(T=0.5,1,5 \mathrm{~K})$. The linear fits shown by the dashed lines demonstrate the power-law dependence of the heating rate on the rf voltage (power-law factor is 6 for $T=0.5 \mathrm{~K}, 5.5$ for $T=1 \mathrm{~K}$, and 4.5 for $T=5 \mathrm{~K}$ ).

character of the quadratic temperature dependence of the rf heating in low-temperature rf-confined plasmas, our data show that there exist different regimes and/or mechanisms for the conversion of the periodic external force into thermal motion. Specifically, the data reported in Ref. [8] show that the heating rate at low temperatures is almost independent of the number of particles in the beam. In our opinion, this means that the heating mechanism responsible for the energy increase is not the Coulomb intrabeam scattering, but instead the scattering on the asymmetric external field (the bending magnetic field in Ref. [9]). It is important to note that in the case of one-dimensional ion string [8], which is much less susceptible to the shear introduced by the bending magnets, the temperature dependence is much sharper than $T^{2}$. The spherical ion cloud studied in Ref. [9] in the radial direction is only subject to a purely quadrupolar harmonic rf field, the same as our linear ion cloud. Therefore, the difference between our results and the temperature dependence reported in Ref. [9] shows that there are different regimes of rf heating. Further studies need to be conducted to determine the factors that affect the rf heating, such as shape and size of the ion cloud, and the trapping parameter $q$.

The change of rf heating rates with trapping voltage is shown in Fig. 3. Three different temperatures of the ion cloud are chosen to study how the heating rates behave in three characteristic regions of the heating curve: the appearance of the rf heating at $0.5 \mathrm{~K}$, the middle of the sharp rise of the heating rate at $1 \mathrm{~K}$, and the middle of the plateau at $5 \mathrm{~K}$. Overall the data indicate that the rf heating rates increase very rapidly with the trapping voltage amplitude. The increase can be approximated by a power-law dependence on rf amplitude with exponent greater than 4 . The power-law dependence with the exponent equal to 4 is predicted by the theory based on Coulomb pair collisions [15]. The pair collision picture is expected to be valid at the higher temperatures, and the power-law exponent for the highest tempera- 
ture in our simulation $(T=5 \mathrm{~K})$ is quite close to that prediction. However, as the temperature decreases, the slope of the power-law curve increases. This indirectly confirms that the applicability of the Coulomb pair collision picture is limited to the higher temperatures.

To obtain insight into the influence of the size of the ion cloud on the rf heating, simulations were also performed for different numbers of particles in the ion cloud. Again, the trapping voltage amplitude $V_{0}=100 \mathrm{~V}$, and the length of the simulated slice is $L=200 \mu \mathrm{m}$. The results are shown in Fig. 4. At $T=5 \mathrm{~K}$ the increase of the rf heating rate with the number of ions is practically linear. This behavior is easy to explain since the average kinetic energy of the micromotion in the ion cloud increases linearly with the number of ions. However, this simple trend is not obvious at the lower temperatures. The $\mathrm{rf}$ heating rate at the lowest temperature shown in the graph $(T=0.5 \mathrm{~K})$ is actually decreasing as the number of ions in the cloud increases, and then exhibits a jump around the linear density that corresponds to a complete shell. At the intermediate temperature $T=1 \mathrm{~K}$ the $\mathrm{rf}$ heating rate shows both a gradual increase, as well as fluctuations correlated with the number of ions in the cloud. It is important to note that at the above temperatures there is no indication of a shell structure in ion radial distribution. However, these $\mathrm{rf}$ heating rate variations indicate preformation of the shell structure and show that some shell structures are more stable than others, e.g., the transitional shell+string configuration is the least stable.

In summary, we have investigated the rf heating rates exhibited by long ion clouds in a linear rf trap. The results show a much sharper increase of the heating rates with tem-

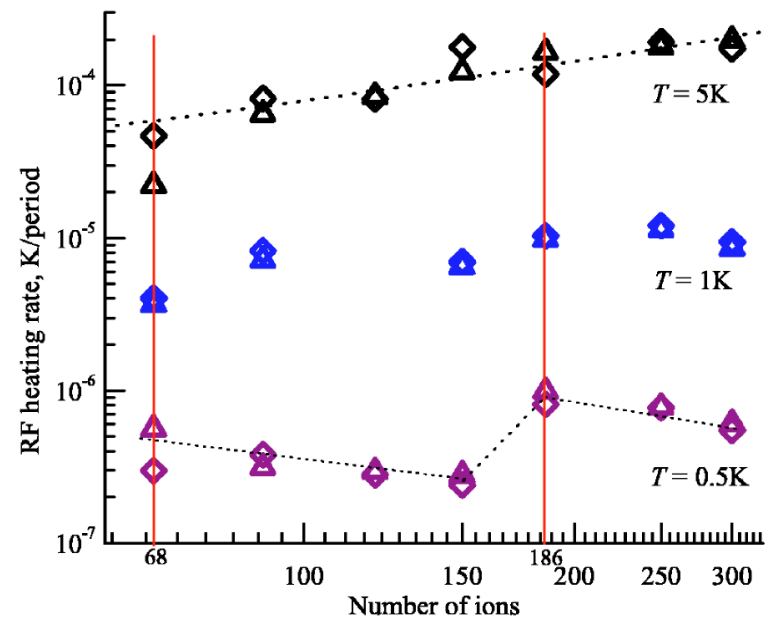

FIG. 4. Change of rf heating rate with the number of particles. Dashed line at $T=5 \mathrm{~K}$ is a linear fit to the data. Dashed line at $T$ $=0.5 \mathrm{~K}$ is given to help identify the shell structure influence. Vertical lines at $N=68$ and 186 indicate the ion clouds that form a "complete shell" in the ion crystal state. Symbols on the plot: $\diamond$, radial motion; $\triangle$, axial motion.

perature in the low-temperature region than was previously reported, which suggests a different rf heating regime. The dependence of the rf heating on the number of ions at low temperature is correlated with the type of the shell structure that is formed at even lower temperatures.

The authors gratefully acknowledge support from the Welch Foundation under Grant No. A-1546.
[1] P. K. Ghosh, Ion Traps (Clarendon Press, Oxford, 1995).

[2] R. Blümel, C. Kappler, W. Quint, and H. Walther, Phys. Rev. A 40, 808 (1989).

[3] J. Prestage, A. Williams, L. Maleki, M. Djomehri, and E. Harabetian, Phys. Rev. Lett. 66, 2964 (1991).

[4] M. Drewsen, C. Brodersen, L. Hornekær, J. S. Hangst, and J. Schiffer, Phys. Rev. Lett. 81, 2878 (1998).

[5] L. Hornekær and M. Drewsen, Phys. Rev. A 66, 013412 (2002).

[6] L. Hornekær, N. Kjærgaard, A. Thommesen, and M. Drewsen, Phys. Rev. Lett. 86, 1994 (2001).

[7] K. Mølhave and M. Drewsen, Phys. Rev. A 62, 011401 (2000).

[8] J. Wei, H. Okamoto, and A. M. Sessler, Phys. Rev. Lett. 80,
2606 (1998).

[9] J. P. Schiffer, M. Drewsen, J. S. Hangst, and L. Hornekær, Proc. Natl. Acad. Sci. U.S.A. 97, 10697 (2000).

[10] N. W. McLachlan, Theory and Application of Mathieu Functions (Dover Publications, New York, 1964).

[11] X. Zhao, V. L. Ryjkov, and H. A. Schuessler, Phys. Rev. A 66, 063414 (2002).

[12] M. P. Allen and D. J. Tildesley, Computer Simulation of Liquids (Oxford Science Publications, Oxford, 1987).

[13] C. Gear, Numerical Initial Value Problems in Ordinary Differential Equations (Prentice-Hall, Englewood Cliffs, NJ, 1971).

[14] Q. A. Turchette et al., Phys. Rev. A 61, 063418 (2000).

[15] T. Baba and I. Waki, Appl. Phys. B: Lasers Opt. 74, 375 (2002). 